\title{
Enhanced spectrum sensing based on Cyclo-stationary Feature Detection (CFD) in cognitive radio network using Fixed \&amp; Dynamic Thresholds Levels
}

Prajwal Patil ${ }^{1}$, Pradeep R Pawar ${ }^{2}$, Praneeth P Jain ${ }^{3}$, Manoranjan K V ${ }^{4}$, Devasis Pradhan ${ }^{5 *}$ (IEEE Member)

${ }^{1-4}$ Final Year U.G Students, Department of Electronics \& Communication Engineering, Acharya Institute of Technology, Bengaluru -
560107 ,Karnataka,India
${ }^{5}$ Assistant Professor, Department of Electronics \& Communication Engineering, Acharya Institute of Technology, Bengaluru -560107, Karnataka, India

DOI: $10.36348 /$ sjet.2020.v05i06.003

| Received: 15.06 .2020 | Accepted: 22.06 .2020 | Published: 25.06 .2020

*Corresponding author: Devasis Pradhan

\section{Abstract}

The advancement in the digital world has led to the increase in the demand for the radio spectrum, which eventually has led to the spectrum scarcity and underutilization of it. The under-utilization of allocated spectrum is due to the fixed spectrum allocation to the primary user. This problem can be overcome by an intelligent radio network called Cognitive Radio (CR). The CR helps in detecting the idle spectrum in the radio environment and thus allocating it to the Secondary Users (SU). Cognitive Radio provides reliable service to the secondary user by preventing interference between secondary and primary user. Spectrum Sensing plays the vital role in the Cognitive Radio to sense the idle spectrum and allocate the vacant spectrum for SU. The major and prominent spectrum sensing methods include Energy Based Detection (EBD), Matched Filter Detection (MFD), Cyclostationary Feature Detection (CFD), Covariance Based Detection (CBD) and Wavelet Based Detection (WBD). This paper discusses Cyclostationary based detection method exploit the periodic statistics and spectral correlation of the received signal to detect the presence of the primary signal in the channel. In wavelet based spectrum sensing wavelet transform technique is applied on the received signal to determine the edges in the spectrum of the signal.

Keywords: Cognitive Radio (CR); Spectrum Sensing; CFD; SCD; FAM; SSCA; SNR.

Copyright @ 2020: This is an open-access article distributed under the terms of the Creative Commons Attribution license which permits unrestricted use, distribution, and reproduction in any medium for non-commercial use (NonCommercial, or CC-BY-NC) provided the original author and source are credited.

\section{INTRODUCTION}

The Rapid increase in the wireless technology and cellular system leads to the development of variety of application which requires sufficient bandwidth. Due to increase in the mobile phone user and IOT (internet of things) radio spectrum becomes more congested. And also due to fixed spectrum allocation there is underutilization of the spectrum. Cognitive radio (CR) emerged as the key technology to solve the problems of Spectrum scarcity and underutilization of spectrum. CR senses the spectrum for the presence of the primary user and allocates the vacant spectrum to the Secondary user. CR dynamically allocates the nearby vacant frequency channel to the secondary user when the primary user needs its channel for data transmission hence cognitive radio provides reliable communication. The various spectrum sensing Techniques in cognitive radio are Energy based detection method, Matched filter detection method, Cyclostationary feature detection method, wavelet based detection method and covariance based detection method.
Energy detection method determines the power of the received signal and compares with it the predetermined Threshold. However energy detection method does not perform well under Low SNR values of the signal. Matched filter detection needs the prior knowledge of the signal and it performs well under Low SNR values because matched filter maximizes the SNR value of the signal. Matched filter senses the spectrum in short time but requires synchronization and some prior knowledge of the primary user such as modulation type, bandwidth etc. The signals used in the digital communication system have special statistical properties such as double sidedness due to sine wave carrier and keying rate due to symbol period. Such signal have special characteristics called Cyclostationary features i.e. the statistical characteristics of such signal vary periodically. This cyclostationarity property can be extracted by using cyclic Autocorrelation. 
Prajwal Patil et al., Saudi J Eng Technol, June., 2020; 5(6): 271-277

Spectrum sensing can be performed in very low SNR by using this method because the noise does not possess periodic statistical characteristics which are random in nature. Hence one can easily distinguish between noise and primary signal. Due to its complexity this technique is not often used. In the wavelet based spectrum sensing method the desired frequency spectrum is divided in sub bands and secondary users are allocated in between the two sub bands. It is also termed as edge detection method in which wavelet transform Technique is employed to determine the edges in the psd of the received signal. The Edges in the frequency spectrum of the signal is due to irregularities of frequencies in different sub bands.

\section{Cognitive Radio}

Cognitive radio (CR) is a radio for wireless communication, which changes their transmission and reception behaviors according to the environment for taking captive of best obtainable spectrum and circumvents inflicting interference on licensed user. It allows an effectual usage of underutilized radio spectrum and also allows sharing of available spectrum by secondary user with primary user. In terminology of $\mathrm{CR}$, the licensed user that accesses a particular spectrum freely which is allocated by the FCC is called primary user (PU). It has higher priority. The unlicensed users, which dynamically exploit the unused frequency, are known a secondary user (SU) and have low priority. The SU must be capable of evaluating the working environment for the existence of PU. Transmission by unlicensed user takes places only if the primary user is not accessing that frequency band. The secondary user should be competent to depart the band or spectrum if transmission by a primary user is recognized. Cognitive radio must have capability and reconfigurability characteristics to make this attainable.

\section{There are following properties of $\mathbf{C R}$}

i) Cognitive capability: CRNs must be capable of selecting a suitable spectrum space by continuous examination of the desired environment or spectrum. This become attainable by spectrum management process, where a number of variables i.e. modulation type, frequency, power, etc., are evaluated. A cognitive cycle is required for the adaptive operation of cognitive capabilities as shown in Figure 1. This involves following operations.

a) Spectrum Sensing: This is a vital part of CR. The presence of $\mathrm{PU}$ and underutilized frequency range is observed by spectrum sensing process. There are local sensing techniques, database registry and beacon signals which make this possible. Beacon signal brings knowledge regarding channel quality as well as spectrum.

b) Spectrum analysis: The characteristics of various channels or networks are analyzed in this section. Then, by using sensing process, unused space is obtained.

c) Spectrum decision: This is the way toward selecting most applicable range of spectrum as indicated by client prerequisite and range qualities for transmission. A single CR or output can make spectrum decision from various CRs.

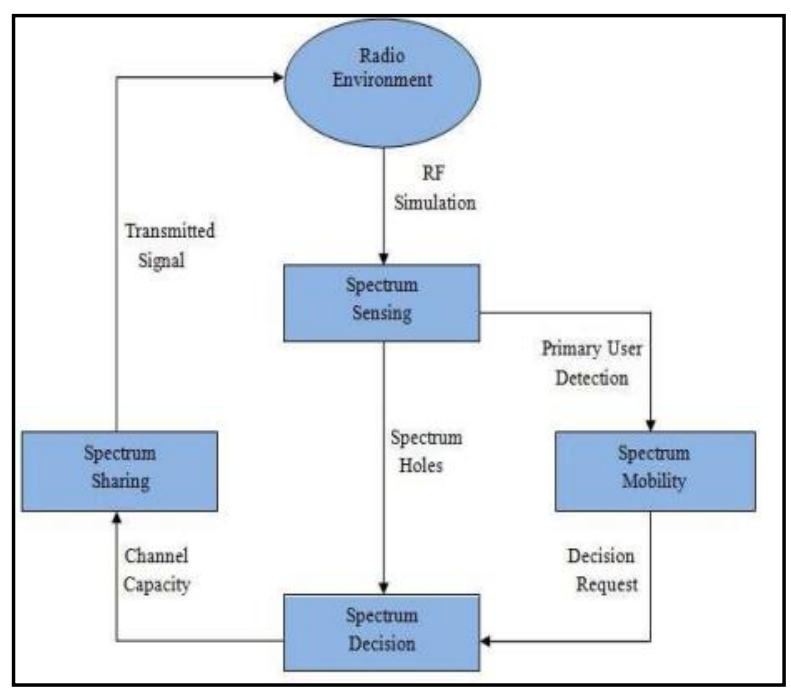

Fig-1: Cognitive Cycle

ii) Reconfigurability: After selecting free space, the next step is to communicate within selected space by changing its several parameters (e.g. type of modulation, frequency and transmission power). This is imperative since CRs, intended to make utilization of spectrum range in a opportunistic way, withdrawing a band if event of PU is seen by utilizing range hand-off technique. Software Defined Radios (SDRs) is used to make this feasible for reconfigurability characteristic

\section{Cyclostationary Feature Detection (CFD)}

Cyclostationary feature detection exploits the Cyclostationary features or properties of the received signal to detect the primary user signal in the spectrum. The Cyclostationary features of the signals have periodic statistics like mean and autocorrelation etc. varies in periodic interval of time. This method has higher immunity to noise in the channel than any other spectrum sensing methods because noise is random in nature and does not have Cyclostationary features. This Cyclostationary features can be extracted from the signal by using parameter called cyclic autocorrelation function (CAC). 


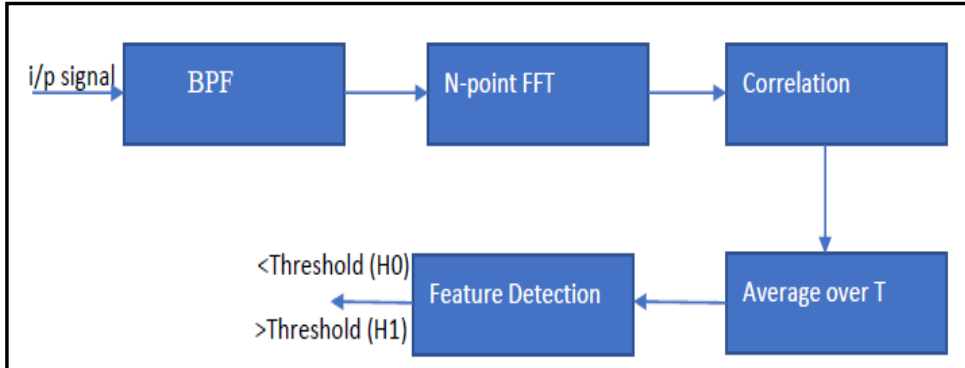

Fig-2: Cyclostationary feature detection method

The cyclic autocorrelation of the received signal is given by the formula;

$$
R_{X}^{\alpha}(\alpha)=\lim _{\Delta \rightarrow \infty} \frac{1}{\Delta} \int_{\frac{-\Delta}{2}}^{\frac{\Delta}{2}} x\left(t+\frac{\tau}{2}\right) x^{*}\left(t-\frac{\tau}{2}\right) e^{-2 \pi \alpha t} d t
$$

$\alpha$ is cyclic frequency, and $\tau$ is time delay, if there is at least one non zero cyclic frequency $\alpha$ such that $\left|R_{X}^{\alpha}\right|$

Must be greater than zero then we say that the received signal $\mathrm{x}(\mathrm{t})$ exhibits Cyclostationary. The cyclic frequency depends on type of Modulation, symbol duration etc. in digital modulated signals of symbol period $T_{b}$ The cyclic frequency exists at integer multiple of reciprocal of $\mathrm{T}_{\mathrm{b}}$. The Fourier transform of the cyclic autocorrelation gives spectral correlation density defined by the equation.

$$
S_{X}^{\alpha}=\int_{-\infty}^{\infty} R_{X}^{\alpha}(\alpha) e^{-i 2 \pi f \tau} d \tau
$$

The received signal $x(t)$ has cyclostationarity i.e. that there exists at least one non-zero cyclic frequency $\alpha$ for all $R_{X}^{\alpha}$ Is not equal to zero for some $\tau$ while the noise is stationary for any non-zero cyclic frequency $\alpha R_{\eta^{\prime}}^{\alpha}$ is equal to zero for all $\mathrm{C}$.

In binary signal detection or spectrum sensing there are two hypothesis $\mathrm{H}_{0}$ signal is absent, $\mathrm{H}_{1}$ signal is present.

The received signal is defined by

$\mathrm{H}_{0}=\mathrm{n}(\mathrm{t})$;

$\mathrm{H}_{1}=\mathrm{x}(\mathrm{t})+\mathrm{n}(\mathrm{t})$;

$\mathrm{n}(\mathrm{t})$ is the noise, $\mathrm{x}(\mathrm{t})$ is the received signal

Let $\alpha_{0}$ be non-zero cyclic frequency for which $R_{X}^{\alpha}$ is not equal to zero for some $C$.assuming the noise and signal are mutually independent we have

$$
H 0: R_{y}^{\alpha 0}(\tau)=0
$$

$H 1: R_{y}^{\alpha 0}(C) \neq 0$ For some $\tau$

Hypothesis $\mathrm{H}_{0}$ and $\mathrm{H}_{1}$ in frequency domain are;

:H0: $S_{X}^{\alpha}(\tau)=0$

$H 1: S_{X}^{\alpha}(C) \neq 0$ For some $\mathrm{f}$

The $\mathrm{H}_{0}$ AND $\mathrm{H}_{1}$ can be distinguished by generating test statistic from the cyclic autocorrelation or spectral correlation density at cyclic frequency $\alpha_{0}$ and comparing the test statistic with the threshold. A typical threshold is given by the equation

$$
C=\int\left|R_{y}^{\alpha 0}(\tau)\right|^{2} d \tau
$$

In practice there are only limited number of samples are available. Let $\mathrm{T}_{\mathrm{s}}$ be the sampling period and $\mathrm{N}$ be the

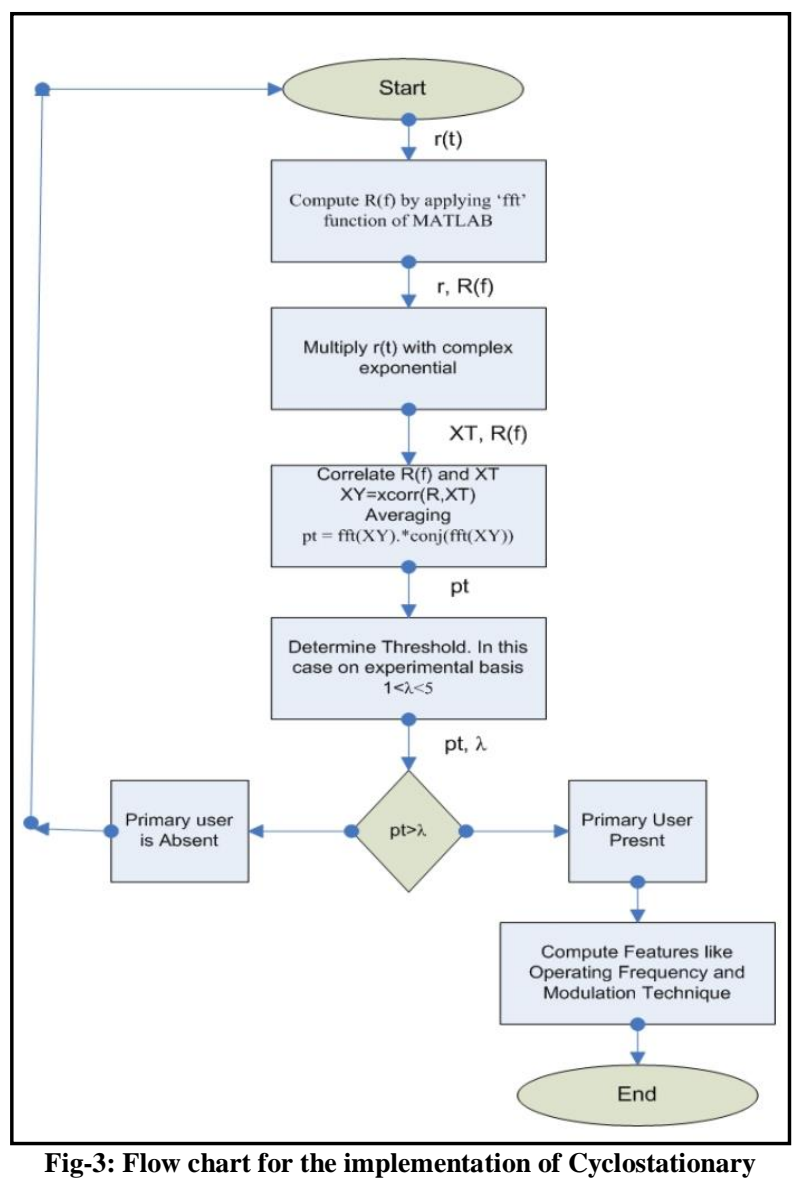
Feature Detection

Number of samples. The discrete version of autocorrelation is given by

$$
R_{Y}^{\alpha}(K T s)=\frac{1}{N} \sum_{n=0}^{M-1} y((n+k) T s) y^{*}(n T s) e^{-2 \pi \alpha n T s}
$$


Prajwal Patil et al., Saudi J Eng Technol, June., 2020; 5(6): 271-277

Where the delay $\mathrm{k}=0,1,2 \ldots . \mathrm{M}-1$ with $\mathrm{M}<<\mathrm{N}$. The discrete version of test statistic is given by

$$
C=\int\left|R_{y}^{\alpha 0}(\tau)\right|^{2} d \tau
$$

The threshold is set based on the white noise power in the channel as it is impossible to detect the accurate noise power in the channel. We can use maximum likelihood Estimation of the noise power. The maximum likelihood

Estimation is given by

$$
\sigma_{n}^{2}=\frac{1}{N} \sum_{n=0}^{N-1}|y(n T s)|^{2}
$$

The Threshold is chosen as $\beta \sigma_{n}^{4}$, where $\beta$ is scalar to meet the predefined probability of false alarm and miss detection. The flow chart precisely represents the executed detection process as shown in Figure 3.

\section{Estimation of the Spectral Correlation Density (SCD)}

Cyclic spectral analysis is used to detect the presence of a signal via the spectral correlation density (SCD) function. To accomplish this goal a series of codes that estimates the SCD function were developed in MATLAB language. Those codes are implementations of two FFT based time smoothing algorithms called the FFT Accumulation Method (FAM) and the Strip Spectral Correlation Algorithm (SSCA).

Cyclic spectral analysis algorithms generally fall into two classes: those that average in frequency (frequency smoothing) and those that average in time (time smoothing). Although both classes of algorithms produce similar approximations to the cyclic spectrum, time smoothing algorithms are considered to be more computationally efficient for general cyclic spectral analysis.

\section{FFT Accumulation Method (FAM)}

FAM is the one of the methods of time soothing algorithm which has good efficiency and computation wise. The basic time soothing algorithm can be formed by evaluating the time soothed cyclic period gram at cyclic frequency $\left(f_{0}, \alpha_{0}\right)$. The Time Smoothed Cyclic Cross Periodogram is given by,

$$
S_{x y}^{\propto 0}(n, f 0)_{\Delta t}=\left[X_{T}\left(N n, f_{0}+\propto_{0} / 2\right) Y_{T}{ }^{*}\left(n, f_{0}-\propto_{0} / 2\right)\right.
$$

The modification in the time soothing algorithm leads to various computation algorithms such as FFT accumulation method (FAM) and Strip spectral correlation algorithm (SSCA). The FAM is a Fourier transform of the correlation product between the spectral components smoothed over time. The SSCA is the Fourier transform of the correlation products between the temporal and spectral components smoothed over time.
FAM consists of capturing in a time length $\Delta \mathrm{t}$ a piece of incoming signal $x[n]$ which is the sampled version of $x[t]$ at $f s$, spectral density is calculated in this range. The computation is performed iteratively over consecutive pieces in the time domain until acceptable result for a summation of several $S_{x}^{\alpha}(n, f)_{\Delta t}$ satisfy the application. In terms of time of computation and objective to meet the FAM cross spectral estimate is given by,

$$
S_{x y}\left(p l, f_{h}\right)_{\Delta t}=\sum X T(r L, f k) Y T^{*}(r L, F 1) g c(p-r) e^{j 2 \pi r q / p}
$$

The FAM Auto spectral estimate is given by,

$$
S_{x x}\left(p l, f_{h}\right)_{\Delta t}=\sum X T(r L, f k) Y T^{*}(r L, F 1) g c(p-r) e^{j 2 \pi r q / p}
$$

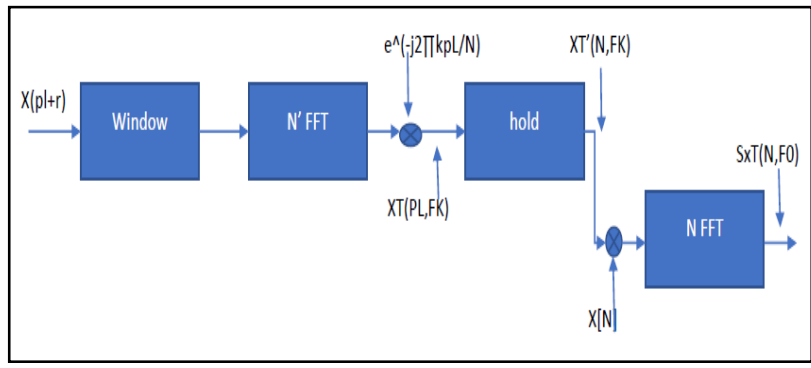

Fig-4: Block Diagram for FAM algorithm

$\mathrm{XT}(\mathrm{rl}, \mathrm{fk})$ and YT (rl, fl) are the complex demodulates inputs for the FFT block and $\mathrm{gc}(\mathrm{p}-\mathrm{r})$ represents the hamming window. The FAM cross spectral plane which is the output of the first FFT has a number of frequencies represented in a 2 dimension plane. Figure 4. Shows the block diagram for FAM.

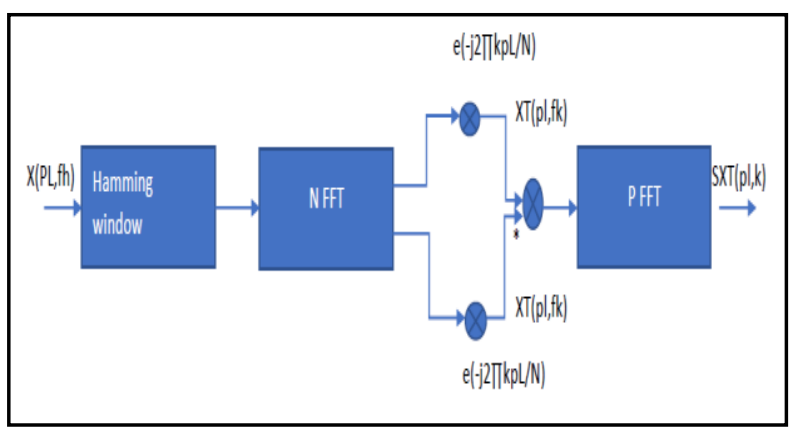

Fig-5: Block diagram of strip spectral correlation algorithm

The FAM is implemented by forming an array from $\mathrm{X}(\mathrm{kT})$, where $\mathrm{k}$ varies from 0 to $\mathrm{N}-1$. Two dimensional arrays with Columns representing constant frequencies are obtained by applying the input to the Hamming Window, Fast Fourier Transformed and then down converted to baseband.

\section{Strip Spectral Correlation algorithm (SSCA)}

The SSCA is the FFT based Time soothing algorithm. The SSCA is implemented by forming an array from $\mathrm{X}(\mathrm{KT})$ with rows $\mathrm{N}$ point long from the input sample data. The starting point of each Succeeding row is offset from the previous rows starting positions from $\mathrm{L}$ samples. The hamming 
Prajwal Patil et al., Saudi J Eng Technol, June., 2020; 5(6): 271-277

window is used across each row which is fast Fourier transformed and converted to baseband. Strip spectral correlation algorithm allows directly multiplying demodulates $\mathrm{XT}(\mathrm{n}, \mathrm{fk})$ with $\mathrm{y}^{*}(\mathrm{n})$. The strip estimate is calculated using the above formula.

The SSCA Cross Spectral estimate is given by,

$$
S\left(n, f_{k} / 2\right)_{\Delta t}=\sum X T(r, f) Y^{*}(r) g c(n-r) e^{j 2 \pi r q / n)}
$$

The SSCA Auto spectral Estimate is given by,

$$
S\left(n, L_{1}, f_{k} / 2\right)_{\Delta t}=\sum X T(r, f) Y^{*}(r) g c(n-r) e^{j 2 \pi r q / n)}
$$

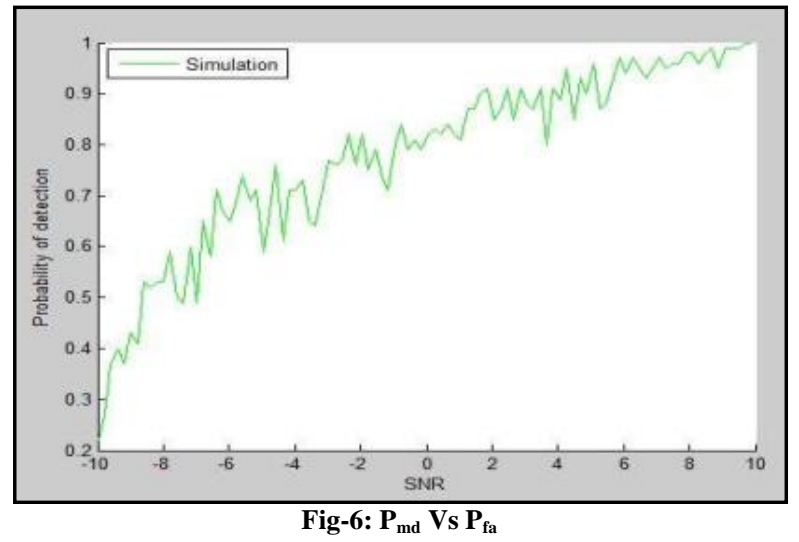

\section{Advantage of Cyclo-stationary Feature Detection Method}

In wireless communication systems' signals can be characterized as cyclostatonary which exhibit underlying periodicities in their structures. These periodicities have a distinctive specific pattern in which that results from the correlation of the signal with the shifted version of itself by a specific value makes signal selectivity possible even at very low SNR. Due to the narrow frequency content along the $(\alpha)$ axis based on transferring from the second order periodicity to first order periodicity.

Consequently, cyclostationary, cyclic frequency and cyclic spectrum are arising from any periodically behaviour of the signal process. In addition richer information is preserves in cyclic spectrum domain such as frequency and phase representation of the incoming signal related to these periodicities. Moreover, from implementation perspective, the important point that interference and noise exhibit no spectral correlation in which that the spectral correlation of the noise is unique and large at cyclic frequency equals to zero comparing to other cyclic frequencies.

\section{RESULTS \& DISCUSSION}

The evaluation of results is done for cyclostationary feature detection and modulation techniques are used as core for spectrum access based performance improvisation for the detected spectrum.In this work, simulation results are analysed for different sample such as $\mathrm{N}=100,200,600,1000$ values but in this paper results are given for $\mathrm{N}=200$ samples and SNR is $-10 \mathrm{~dB}$ (tested range from $-10 \mathrm{~dB}$ to $10 \mathrm{~dB}$ ).

a) Probability of miss detection $\left(P_{m d}\right)$ and probability false alarm $\left(\mathbf{P}_{\mathbf{f a}}\right)$ : Probability of miss detection is the probability of missing the detection or missing the PU while detecting the spectrum. It should be less as much as possible and probability of false alarm is the probability when the PU is detected by mistake i.e. there is no primary user in the desired spectrum but it is detected by the system. It should be low for the better performance of the system and for better outcomes. Figure 6. Shows the Pmd vs Pfa curve for 200 samples. This curve shows that probability of false alarm and probability of miss detection are inversely proportional to each other. The Pmd is 0.47 , $0.33,0.29,0.24,0.21,0.09,0.09,0.03,0.02$ and 0 for Pfa value $0.1,0.2,0.3,0.4,0.5,0.6,0.7,0.8$ and 0.9 . The mean Pmd is 1.77 . if probability of false alarm increases, probability of miss detection decreases. Hence, these two parameters cannot decrease simultaneously.

b) Probability of detection (Pd): It is the time during which licensed user or primary user (PU) is being detected or recognized in a given spectrum. It is a fundamental part for the system performance or output analysis. When PU is accesing then SU cannot access. Figure 7 below shows the signal to noise ratio Vs probability of detection curve for 200 samples. The range of the SNR is from $-10 \mathrm{~dB}$ to $+10 \mathrm{~dB}$. This curve shows that the SNR and probability of detection are directly proportional to each other. With increase in $\mathrm{SNR}$, the Pd is also increases. The detection probability for SNR -10 to 10 is $0.22,0.532,0.699,0.657,0.807$, $0.815,0.867,0.951,0.98$ and 1 . It is analysed that cyclostationary feature detction has high probability of detection.

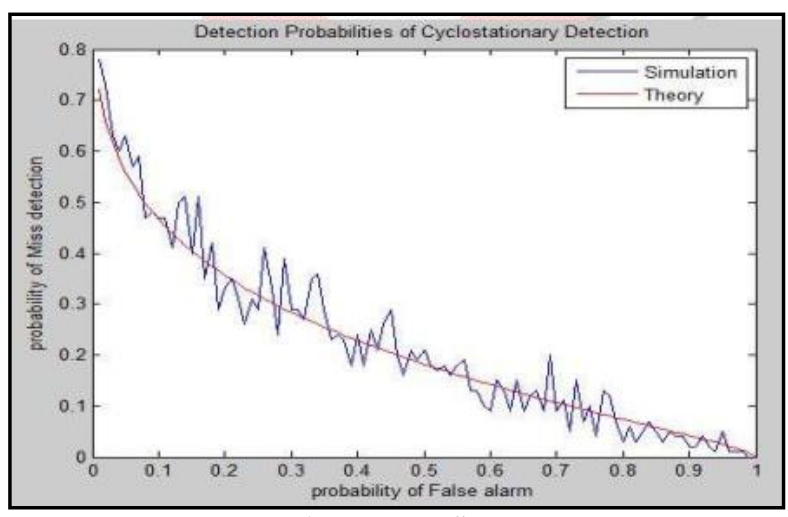

Fig-7: Pd Vs SNR

\section{Comparison between the dynamic and fixed threshold}

To set a fixed threshold, the constant false alarm rate principle (CFAR) is used, in which the probability of false alarm $(P f a)$ is set to 0.1 . Thesimulation is done for a fixed number of samples $\mathrm{N}$ 
$=5000$. Figure 8 and Figure 9 show a comparison between the fixed and dynamic threshold setting for CSFD in terms of $P d$ and $P f a$, respectively. These results show that the dynamic threshold decreases the misdetection probability and increases the false alarm probability. Therefore, it can be concluded that there is no way to improve one of these probabilities without compromising the other one. Determining which probability must be restricted depends on user's preferences and CR's application. In other words, if it is very important to not miss any frequency opportunity, probability of false alarm must be constrained to be as small as possible to guarantee high spectrum utilization.

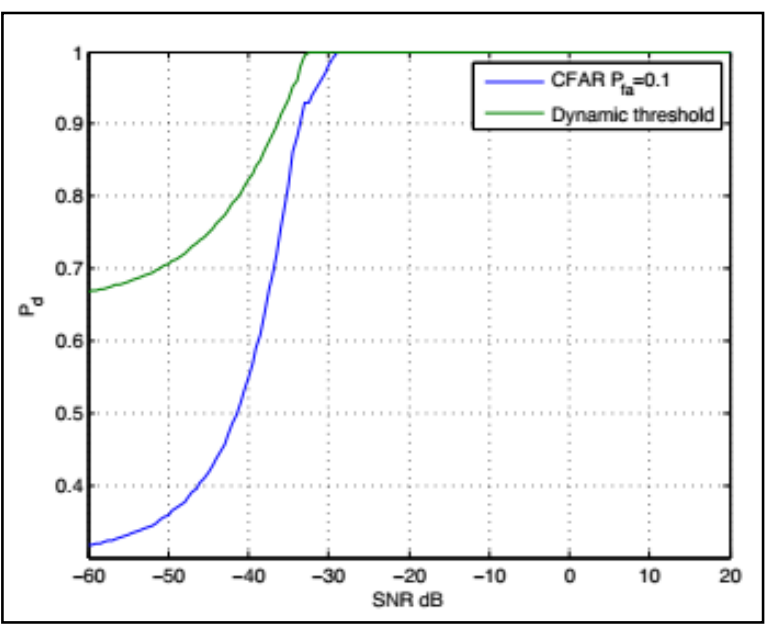

Fig-8: $P_{d}$ for fixed and dynamic threshold under different SNR

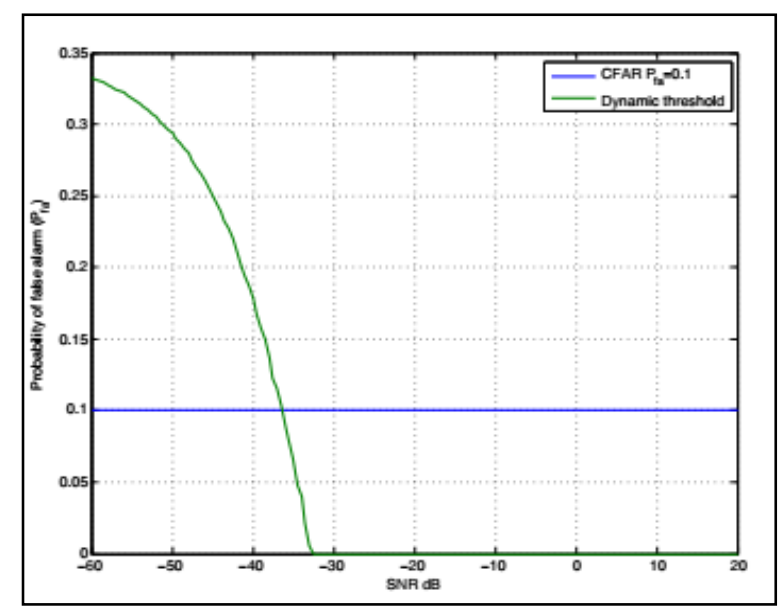

Fig-9: $P_{f a}$ for fixed and dynamic threshold under different SNR

\section{CONCLUSION}

In this paper Cyclostationary Feature Detection method was discussed and implemented in the Matlab software. The spectrum sensing can be performed more reliably using this method. This method has high computation complexity but it has higher noise immunity than other spectrum sensing methods. From the simulation result we inferred that this method has better spectrum sensing results compared to the energy and matched filter detection method.

\section{ACKNOWLEDGEMENT}

Firstly, we highly indebted to the Department of Electronics \& Communication Engineering for giving us the opportunity to complete this research work. We sincerely express our sincere gratitude and deepest appreciation to Dr. Rajeswari, Professor, HoD of ECE and Principal of Acharya Institute of Technology, Bangalore, Karnataka, for guiding and encouraging us to develop and simulate the control designs essential for this work and support. We would like thank to Mr. Devasis Pradhan for guiding us throughout the course of our project through which the research work come into a streamline work and we also thankful to our beloved parents for their trust on us and their believe.

\section{REFERENCES}

1. Aparna, P. S., \& Jayasheela, M. (2013). Cyclostationary feature detection in cognitive radio for ultra-wideband communication using cooperative spectrum sensing. International Journal of Future Computer and Communication, 2(6), 668.

2. Subhedar, M., \& Birajdar, G. (2011). Spectrum sensing techniques in cognitive radio networks: A survey. International Journal of Next-Generation Networks, 3(2), 37-51.

3. Ganesan, G., \& Li, Y. (2007). Cooperative spectrum sensing in cognitive radio, part II: multiuser networks. IEEE Transactions on wireless communications, 6(6), 2214-2222.

4. Sahai, A., Mishra, S. M., Tandra, R., \& Woyach, K. A. (2009). Cognitive radios for spectrum sharing [applications corner]. IEEE Signal processing magazine, 26(1), 140-145.

5. Tani, A., \& Fantacci, R. (2010). A low-complexity cyclostationary-based spectrum sensing for UWB and WiMAX coexistence with noise uncertainty. IEEE Transactions on Vehicular Technology, 59(6), 2940-2950.

6. Haykin, S. (2005). Cognitive radio: brainempowered wireless communications. IEEE journal on selected areas in communications, 23(2), 201-220.

7. Mohammadi, A., Taban, M. R., Abouei, J., \& Torabi, H. (2013). Cooperative spectrum sensing against noise uncertainty using Neyman-Pearson lemma on fuzzy hypothesis test. Applied Soft Computing, 13(7), 3307-3313.

8. Aparna, P. S., \& Jayasheela, M. (2012). Cyclostationary feature detection in cognitive radio using different modulation schemes. International Journal of Computer Applications, 47(21).

9. Duan, D., Yang, L., \& Principe, J. C. (2010). Cooperative diversity of spectrum sensing for cognitive radio systems. IEEE transactions on signal processing, 58(6), 3218-3227.

10. Hwang, J., \& Kim, J. (2011). U.S. Patent No. 7,864,830. Washington, DC: U.S. Patent and Trademark Office. 
Prajwal Patil et al., Saudi J Eng Technol, June., 2020; 5(6): 271-277

11. Chen, C. Y., Tseng, F. H., Chang, K. D., Chao, H. C., \& Chen, J. L. (2010). Reconfigurable software defined radio and its applications. Tamkang Journal of Science and Engineering, 13(1), 29-38.

12. Fragkiadakis, A. G., Tragos, E. Z., \& Askoxylakis, I. G. (2012). A survey on security threats and detection techniques in cognitive radio networks. IEEE Communications Surveys \& Tutorials, 15(1), 428-445.

13. Mahajan, R. (2015, September). Performance analysis of cyclostationary and energy detection spectrum sensing techniques. In 2015 International Conference on Signal Processing, Computing and Control (ISPCC) (pp. 247-251). IEEE.

14. Rebeiz, E., Urriza, P., \& Cabric, D. (2013). Optimizing wideband cyclostationary spectrum sensing under receiver impairments. IEEE Transactions on signal processing, 61(15), 39313943.

15. Mahmoud, H. A., Yucek, T., \& Arslan, H. (2009). OFDM for cognitive radio: merits and challenges. IEEE wireless communications, 16(2), 6-15.

16. Tarique, M. (2016). Performances of orthogonal wavelet division multiplex (owdm) system under awgn, rayleigh, and ricean channel conditions. International Journal of Computer Networks \& Communications (IJCNC), 8(3).

17. Devasis, P., \& Priyanka, K.C. (2019). "Effectiveness of Spectrum Sensing in Cognitive Radio toward 5G Technology", Saudi Journal of Engineering and Technology, Dec. 4(12): 473-785. 